\title{
Selective amotivation deficits following chronic psychosocial stress in mice
}

Alejandro Tsai Cabal ${ }^{1,2,3, \S}$, Horea-loan loanas ${ }^{1,2,4}$, Erich Seifritz , $^{1,2,5}$ and Bechara J. Saab ${ }^{1,2,5 *}$

${ }^{1}$ Preclinical Laboratory for Translational Research into Affective Disorders, DPPP, Psychiatric Hospital, University of Zurich, August-Forel-Strasse 7, CH-8008 Zurich, Switzerland

${ }^{2}$ Neuroscience Center Zurich, University of Zurich and ETH Zurich, Switzerland

${ }^{3}$ Department of Biology, ETH Zurich, Switzerland

${ }^{4}$ Institute for Biomedical Engineering, University and ETH Zurich, $\mathrm{CH}-8093$, Zurich, Switzerland

${ }^{5}$ Department of Psychiatry, Psychotherapy and Pesychosomatics (DPPP); Psychiatric Hospital, Universit of Zurich, Lengstrasse 31, CH-8032 Zurich, Switzerland

${ }^{\S}$ Present address: Friedrich Miescher Institue (FMI) for Biomedical Research, Maulbeerstrasse 66, P.O. Box 3775, CH-4002 Basel, Switzerland

${ }^{*}$ Corresponding Author:

PI Dr Bechara J Saab

Platrad, University of Zurich Hospital for Psychiatry

August Forel Strasse 7

$\mathrm{CH}-8008$, Zurich

Switzerland

+41446353364

bechara.saab@uzh.ch 
Abbreviations

CSD: Chronic social defeat

MDD: Major depressive disorder

EPM: Elevated plus maze

NFT: New frontier task 


\section{Abstract}

Amotivation is a major symptom of several psychiatric disorders. However, which specific motivations are most affected in various illnesses is not well understood. In major depressive disorder (MDD), anecdotal evidence suggests the motivation to explore may be especially affected, but direct evidence from either patients or animal models is lacking. To investigate the potential for, and nature of, exploratory drive deficits in MDD, we subjected mice to a chronic social defeat (CSD) manipulation that gives rise to a MDD-like behavioural ensemble, and performed a behavioural battery to examine bodyweight homeostasis, ambulation, anxiety, exploratory behaviour motivated by either novelty or fear, and short-term memory. Consistent with previous reports, we found a disruption of bodyweight homeostasis and reduced ambulation following CSD treatment, but we found no evidence for anxiogenic effects or impairments in short-term memory. Surprisingly, we also observed profoundly delayed and diminished exploration of novel, safe space following CSD, while exploration motivated by fear remained intact. These results extend our knowledge of the behavioural phenotypes in mice resulting from CSD by homing in on specific motivational drives. In MDD patients, reduced exploration could compound disease symptomatology by preventing engagement in what could be rewarding exploration experiences, and targeting deficits in the motivation to explore may represent a novel avenue for treatment. 


\section{Keywords}

Curiosity, Major Depressive Disorder (MDD), New Frontier Task (NFT), Spatial Memory, Exploration, Mice 


\section{Introduction}

Amotivation is a symptom of many mental illnesses, and a core symptom of major depressive disorder (MDD) (Calabrese et al., 2014; DSM-5, 2013). While amotivation is often considered as a single phenotype, it can probably be more accurately described as a composite of motivational deficits, all of which may not be uniformly affected in a given mental illness. The specific loss of motivation to explore, for example, has been reported in some mental illnesses that exhibit cognitive dysfunction, such as autism (Williams et al., 1999; Gustafsson \& Paplinski, 2004) and schizophrenia (Torgersen et al., 1993; Strauss et al., 2011). In MDD, deficits in exploratory excitability (a measure of novelty-seeking) are prominent, despite no change in overall impulsivity (another measure of novelty-seeking) (Bensaeed et al., 2014; Zaninotto et al., 2016), suggesting patients with MDD experience less reward when presented with novel stimuli. In addition, patients with MDD are less engaged by, and are less engaging in, their environments (Der-Avakian \& Markou, 2012; Calabrese et al., 2014). Together, these findings hint towards the potential for exploratory drive deficits in MDD. However, little clinical or preclinical research has directly examined exploratory drive in MDD or in experimental animals exposed to MDD-phenotypeinducing manipulations. Understanding if deficits in exploratory drive are present in MDD is important because exploration can yield rewarding information from the environment and facilitate the efficiency of learning (Mun et al., 2015), indicating that restoring the motivation to explore could be a potent new treatment strategy in psychiatry, or at least be a marker for treatment response.

Despite the clear importance of an active exploratory drive in maintaining mental health, exploratory drive deficits have received surprisingly little attention from the clinical and research communities. There is therefore only a limited theoretical framework and narrow selection of tools currently available to probe the mechanisms underlying loss of exploratory drive in mental illness (Der-Avakian \& Markou, 2012; Calabrese et al., 2014; Hershenberg et al., 2016). Given this minimal examination, and since better understanding exploration deficits could yield novel targets 
and strategies for treating mental illness, we examined exploratory behaviours in mice following chronic social defeat (CSD), a social manipulation that induces pathophysiology reminiscent of MDD (Krishnan et al., 2007; Yan et al., 2010; Azzinnari et al., 2014; Fuertig et al., 2015; Grandjean et al., 2016; Bergamini et al., 2016).

To distinguish between different drives to explore, we made use of the new frontier task (NFT), a paradigm that provides experimental animals with the opportunity to climb out from their homecage and explore four dimly lit novel platforms positioned along the cardinal axes (Tsai Cabal et al. 2016; Saab et al., 2009). Classically, the NFT is performed with the homecage either dimly or brightly lit. The dimly lit or dark version of the NFT is proposed to measure volitional exploration motivated by the inherently rewarding nature of novel stimuli, while the brightly lit version is proposed to assess fear-driven exploration, since bright light is a fear-inducing stimulus for small nocturnal prey, like mice. In both types of illumination, the unfamiliar environments (or "frontiers") remain dim. In addition, scents of other mice are eliminated and subjects always have free access to food and water, thereby minimizing potential interference from socio-sexual-satiation drives.

In this study, the NFT was one component of a behavioural battery that also assessed bodyweight homeostasis, ambulation, anxiety and short-term memory. We find strong effects on bodyweight homeostasis and exploration, but no effect on anxiety and short-term memory. With respect to exploration, CSD-treated mice exhibited impairment exclusively under dim illumination, suggesting a surprising specificity with their amotivation phenotype.

The results highlight exploration deficits in a social stress manipulation that induces some important behaviours resembling MDD core symptomatology. The data thereby provide an important starting point for careful interrogation of circuits and molecules involved in the pathogenesis of diminished exploration affecting patients with MDD and other mental illnesses, and highlight exploratory drive as a potential new target for novel treatment strategies in MDD. 


\section{Materials and Methods}

\subsection{Experimental subjects and ethical standards}

All experiments and manipulations conformed to the guidelines set by the Animal Care

Commission of Switzerland and were covered under the authority of animal permit ZH170/2012. All possible measures were taken to ensure minimal pain and discomfort.

Experimental subjects were 24 young adult ( 8 wks of age at study onset) $\mathrm{B} 6 / \mathrm{J}-\mathrm{Rj}$ male mice obtained from Janvier (France). Prior to engaging in any experimental paradigms, the mice were provided with one week to acclimatise to the facility, followed by $10 \mathrm{~min} /$ day of handling for 4 days (Fig. 1A). Mice were maintained on a reverse light cycle (lights off at 7:00 am; lights on at 7:00 pm) with access to Purina mouse chow and sterile water ad libitum throughout the study. Internally ventilated cages containing tissue for nest building, pinewood bedding and a transparent plastic red house were used. The subjects were divided equally into 2 groups: 12 CSD, 12 controls.

Aggressor subjects required to induce CSD consisted of 12 adult ( 8 mth of age) male CD-1 exbreeder mice, pre-selected for demonstrating aggressive behaviour as previously reported (Azzinnari et al., 2014).

\subsection{Chronic social defeat (CSD)}

The two groups, each consisting of 12 mice (CSD or control) were formed by counter-balancing motor activity measured for $15 \mathrm{~min}$ as horizontal and vertical beam breaks in a TSE Multiconditioning system arena (50 lx) one day prior to the CSD manipulation. CSD procedures were then carried out as previously reported (Azzinnari et al., 2014) in a dimly lit room (20 lx) adjacent to the vivarium. For 15 days, CSD mice were introduced daily into the cage of a different aggressive CD-1 mouse for a total of $10 \mathrm{~min}$, or $60 \mathrm{~s}$ of one-to-one attack (cumulative), whichever came first. Subject weights were determined immediately prior to the attack session. The control group was caged in pairs and transferred to new cages on the same days the CD-1 mice received new cages. Bedding was changed by the experimenter on day 7 and 15 during CSD, and at the same 
time of day for both groups. The incisor teeth of CSD mice were trimmed on every third day to minimise the risk of bite wounds and thereby increase the validity of CSD as s psychosocial stressor (Azzinnari et al., 2014).

\subsection{Behavioural testing}

The behavioural paradigms used to compare CSD and control groups were performed sequentially on the same cohort of animals, as shown in figure 1A. Experimental paradigms were conducted in order of least to most aversive. Where possible, all equipment used to test mice was cleaned prior to testing each subject with dry tissues to remove faeces and urine (if present) and with $70 \%$ ethanol to remove potentially interfering odours. A minimum of $48 \mathrm{~h}$ was provided between any two behavioural paradigms, and all experiments were performed during the subjects' active cycle and not within $2 \mathrm{~h}$ of day/night transition times.

\subsubsection{Activity test}

Subjects were placed into a TSE Multi-conditioning system measuring 45 x 45 x $40 \mathrm{~cm}$ (W x D x H) and consisting of a metallic grid cased within PVC housing (Pryce et al., 2012). Total path, rearing events (measured by $5 \mathrm{~cm}$ high infrared beam breaks) and immobility were recorded automatically for $15 \mathrm{~min}$.

\subsubsection{Elevated plus maze}

A Plexiglas plus maze, elevated $50 \mathrm{~cm}$ above the ground, with 2 closed (15 lx) and 2 open (600 lx) arms, each measuring $66 \mathrm{~cm}$ long and $6 \mathrm{~cm}$ wide, was used. Two goose lamps were placed facing the centre of the maze at the end of each open arm, and the entire behavioural suite was illuminated with 6 overhead red lights. No salient extra-maze spatial cues were visible to the mice apart from the goose lamps. The central area of the maze included the cross-sectional area of all four arms. Subjects were placed in the centre of the maze and facing the open arm opposite from the 
experimenter. Activity was recorded for 5 min using an overhead camera and automated tracking system (HVS Image 2015, UK).

\subsubsection{New frontier task (NFT)}

Protocol and conditions were as reported previously (Tsai Cabal et al., 2016). A custom-made set of four planar T-shaped Plexiglas platforms $(18 \mathrm{~cm}$ high, $25 \times 20 \mathrm{~cm})$ were placed around the homecage. A small ladder $(15 \mathrm{~cm}$ high, $5 \times 15 \mathrm{~cm})$ fixed to each platform, protruded into the homecage to facilitate volitional entry to and exit from the new frontiers. Exploratory events, defined as the presence of all four paws on a given platform, were recorded manually. The latency to the first exploration event was recorded and each trial then proceeded for a further $15 \mathrm{~min}$. In the absence of exploratory events during the first $30 \mathrm{~min}$, the trial was stopped after waiting an additional $15 \mathrm{~min}$ (one occurrence in this study: CSD-treated subject under red illumination).

Three different illumination levels were used in order to examine novelty-driven and fear-driven exploration separately. i) Red Lighting (homecage: 15 lx) was used to provide a safe environment and the entire behavioural arena and experimental setup were exclusively illuminated with six equally spaced overhead red LEDs. ii) Bright Lighting (homecage: 1200 lx) was used to provide an unconditioned fearful stimulus and employed a strong white halogen spotlight hanging $12 \mathrm{~cm}$ above the homecage. The halogen spotlight was encased in a rectangular lampshade that prevented light from illuminating the platforms. During the Bright Lighting condition, overhead red LEDs were left on. iii) Minimal Lighting (homecage: 100 lx) was used as a control for the presence of white light, and was identical to the Bright Lighting condition, except the white halogen spotlight was replaced with a $40 \mathrm{~W}$ white incandescent bulb. In all three lighting conditions, the "new frontiers" of the NFT apparatus were illuminated almost exclusively from the overhead red lights and were $\approx 10 \mathrm{~lx}$.

Each subject was given a unique marking on his tail using a felt pen to allow rapid identification by an observer. 


\subsubsection{Hole board task}

Protocol and conditions were as reported previously (Saab et al., 2009). Briefly, a topless, bottomless and colourless Plexiglas frame $\left(40 \mathrm{~cm}^{3}\right)$ was placed over a separate sheet of colourless Plexiglas containing 4 centrally located holes $(1 \mathrm{~cm}$ in diameter $)$ with their centres forming a 25 $\mathrm{cm}^{2}$ square elevated $8 \mathrm{~mm}$ above a second Plexiglas sheet containing no holes. The behavioural suite was illuminated with 3 dimmed white lights (10 lx). Subjects were cupped in hand and gently placed in the centre of the arena and allowed to explore for 5 min before being returned to their homecage. Hole exploration or "head dips" (each of the 4 holes recorded separately, and then combined for analysis), rearing (assisted and unassisted recorded separately, and then combined for analysis), grooming (head and body recorded separately, and then combined for analysis), walking (within $5 \mathrm{~cm}$ from wall or in centre, recorded separately and combined for analysis), risk assessment (no instances observed) and still behaviour (immobility) were recorded manually with Noldus Observer XT software (Netherlands).

\subsubsection{Displaced and Novel Object recognition test}

Protocol and conditions were as reported previously (Saab et al., 2009). Briefly, a topless, bottomless and colourless Plexiglas frame $\left(40 \mathrm{~cm}^{3}\right)$ was placed directly over a separate Plexiglas sheet on which 3 identical objects stood. Two different types of stainless steel objects were used (a $2 \mathrm{~cm}$ diameter sphere mounted on a flat pedestal, total object $3 \mathrm{~cm}$ tall; and a $1 \mathrm{~cm}$ wide $\mathrm{x} 3 \mathrm{~cm}$ tall cylinder), counter-balancing the familiar and novel objects. The behavioural arena was illuminated by 6 equally spaced overhead red lights and two diffuse white lights from underneath $(\approx 20$ lx inside the Plexiglas box).

Object exploration (each object separately, combined for analysis), rearing (assisted and unassisted recorded separately, and then combined for analysis), grooming (head and body recorded separately, and them combined for analysis), walking (within $5 \mathrm{~cm}$ from wall or in centre, recorded separately, and combined for analysis), risk assessment (no observations recorded) and immobility 
were recorded manually with Noldus Observer XT software (Netherlands). Extra-maze visual cues were placed within the behavioural suite on each of the 4 walls surrounding the apparatus, about 1.5 $\mathrm{m}$ away from the Plexiglas walls.

The test was divided into 3 phases separated by 2 min interphase intervals: Phase $1=$ Habituation (15 min); Phase 2 = Displaced object recognition (5 min); Phase 3 = Novel object recognition (5 $\mathrm{min})$.

\subsection{Statistical analysis}

\subsubsection{Bodyweight variability}

Measured daily, binned across 5 day blocks and analysed with a parametric two-tailed unpaired ttest.

\subsubsection{Activity assay}

Horizontal ambulation (total $\mathrm{x}, \mathrm{y}$ beam breaks), duration of immobility (no beam breaks for $>2 \mathrm{~s}$ ) and rearing events (minimum of 6 consecutive vertical beam breaks) pre- and post-CSD were analysed using a repeated measures two-way ANOVA followed by a Bonferroni multiple correction test.

\subsubsection{EPM}

Duration of time spent and pathlength in the closed, open arms and central area of the maze were analysed using a parametric two-tailed unpaired t-test followed by a Bonferroni multiple correction test.

\subsubsection{NFT}

Binned and total exploration event data, as well as latencies to explore, were analysed using a repeated measures two-way ANOVA followed by a Bonferroni multiple correction test. 


\subsubsection{HB}

Behaviour was analysed using a parametric two-tailed unpaired t-test followed by a Bonferroni multiple correction test.

\subsubsection{DONOR}

Behaviour was analysed using a parametric two-tailed unpaired t-test followed by a Bonferroni multiple correction test.

\subsubsection{Asterisks and hashtags}

Asterisks (*) represent $P$ values for between group comparisons as follows: $* * * * P<0.0001$; $* * * P<0.001 ; * * P<0.01 ; * P<0.05$. Hashtags (\#) represent $P$ values for within-group comparisons as follows: \#\#P<0.01; \#P<0.05. Individual data points and/or mean plus $S E M$ are presented. 


\section{Results}

\subsection{CSD reduces bodyweight homeostasis, ambulation and rearing}

Experimental paradigms and manipulations were conducted in the order illustrated (Fig. 1A). Since reduced bodyweight homeostasis during CSD is a robust phenotype, even though its aetiology remains unknown and unexplored, we weighed the CSD and control subjects and analysed daily fluctuation. Consistent with previous reports (Azzinnari et al., 2014; Fuertig et al., 2015; Grandjean et al., 2016), we observed greater bodyweight fluctuations during the CSD manipulation relative to control subjects not treated with CSD (Fig. $1 \mathrm{~B} ; t=5.545, P=0.0001$ ), indicating CSD-induced stress was affecting physiology and/or behaviour.

Next, since changes in anxiety could affect performance in the NFT and anxiogenesis is sometimes observed in defeated mice (Blanchard et al., 2001; Haque et al., 2012; Venzala et al., 2013; Kovalenko et al., 2014; Brachman et al., 2015; Krishnan et al., 2007), we examined the level of unconditioned anxiety in CSD-treated mice using the elevated plus maze (EPM) (Ennaceur, 2014). However, no significant difference was found in the time spent by control and CSD-treated animals in the open or closed arms of the maze (Fig. 1C; open arms: $t=0.616, P=0.5403$, closed arms: $t=0.088, P=0.9302)$. Instead, both groups spent significantly less time in the open compared to closed arms (Fig. $1 \mathrm{C}$; control: $t=3.164, P=0.0028$, CSD: $t=2.528, P=0.0151$ ), indicating the brightly lit open arms of the EPM were aversive for both groups, and the CSD manipulation used here did not affect detection or avoidance of bright lights in a stressful novel environment. At the same time, we did detect a significant difference in pathlength within the closed arms, where the CSD-treated group showed less ambulation (Fig. 1D; $t=2.795, P=0.0106)$. In contrast, ambulation in the brightly lit open arms was not affected by CSD (Fig. 1D; $t=1.165, P=0.2565$ ). We suspect the pathlengths of both groups was larger in the open arms because the mice tended to remain comparatively still in the sheltered parts of the maze.

We then examined ambulation in a dimly lit closed arena (activity assay), since reduced horizontal activity is a major hallmark phenotype of defeated mice (Blanchard et al., 2001; Haque et 
al., 2012; Venzala et al., 2013; Azzinnari et al., 2014; Kovalenko et al., 2014; Brachman et al., 2015; Fuertig et al., 2015). Here we found a significant main effect of the CSD manipulation on horizontal activity $(F(1,22)=6.496, P=0.0183)$ and immobility $(F(1,22)=4.905, P=0.0374)$, and for the CSCxTime interaction on rearing $(F(1,22)=8.202, P=0.0090)$. Bonferroni multiple corrections revealed reduced horizontal ambulation (Fig. 1E; $P=0.0051$ ), increased immobility (Fig. 1F; $P=0.0108)$ and reduced rearing (Fig. $1 \mathrm{~F}, P=0.0068$ ) relative to controls. Within subject comparisons showed an increase in horizontal ambulation amongst the control group (Fig. 1E, $P=0.0298$ ) and decreased rearing in the CSD-treated group (Fig. 1F, $P=0.0435$ ), which is an important exploratory behaviour (Lever et al., 2006; Saab et al., 2009; Mun et al., 2015), following the 15-day CSD period.

These results confirm that the CSD manipulation gave rise to abnormal behaviours and physiology consistent with previous findings (Haque et al., 2012; Azzinnari et al., 2014), and hint towards a previously unreported deficit in exploratory drive.

\section{CSD reduces exploration of novel, safe space}

As CSD is widely used to induce behavioural phenotypes in rodents that are reminiscent of MDD in humans (Yan et al., 2010), and humans with MDD exhibit amotivation and deficits in exploratory excitability (Bensaeed et al., 2014; Calabrese et al., 2014; Zaninotto et al., 2016), we examined both novelty-driven and fear-driven exploration in CSD-treated and control subjects using the NFT (Fig. 2A).

Interestingly, we found a significant main effect of CSD for the latency to explore the new frontiers (Fig. 2B-D). This was true for the latency to visit the first frontier (Fig. 2B; $F(1,22)=9.578$, $P=0.0053$ ), the second frontier (Fig. $2 \mathrm{C} ; F(1,22)=31.76, P<0.0001)$ and all four frontiers (Fig. 2D; $F(1,22)=38.41, P<0.0001)$. Moreover, post-hoc Bonferroni multiple comparison-corrected t-tests revealed increased latencies for CSD-treated subjects to visit the frontiers when the homecage was illuminated with red or dim white lighting (Fig. 2B,C), while no difference was found when the 
homecage was illuminated with bright light (Fig. 2D), suggesting specificity within the motivational deficit.

Once the subjects had visited at least one frontier, we tracked all subsequent exploration events for a further $15 \mathrm{~min}$ and found a significant main effect of CSD on frontier visits in the dark $(F(1,22)=12.20, P=0.0021)$ that was primarily driven by significant differences in the first two binned periods of testing (0-5 min: $P=0.0002 ; 5-10 \mathrm{~min}: P=0.0238)$. A similar result was found under dim homecage illumination $(F(1,22)=16.39, P=0.0010)$, although here the main effect was driven by significant differences throughout testing $(0-5 \mathrm{~min}: P=0.0042 ; 5-10 \mathrm{~min}: P=0.0113 ; 10-$ 15 min: $P=0.0212$ ). In contrast, we did not find a significant main effect of CSD on exploration events when the homacage was brightly illuminated $(F(1,22)=3.767 P=0.1923)$. Finally, the total number of exploration events revealed a significant main effect for $\operatorname{CSD}(F(1,22)=26.72 P=0.0006)$, and Bonferroni corrected post-hoc t-tests revealed significance in the dark $(P=0.0003)$ and dim $(P=0.0009)$ lighting condition, but not under bright illumination $(P=0.9298)$.

To compliment the NFT and compare the results to a more traditional exploration paradigm, we performed the hole board test (File \& Wardill, 1975; Karl et al., 2003). Consistent with the results from the post-CSD activity assay, we found CSD-treated mice spent more time immobile $(t=3.075$, $P=0.0027$ ), while other individual behaviours remained unchanged (Fig. 3A). There was no observable difference in the number of head dips (Fig. 3B; $t=0.296, P=0.7704$ ) however, which has previously been used as a measure of both exploration (File \& Wardill, 1975) and anxiety (Takeda et al., 1998) in this paradigm. Finally, the level of grooming, which is sometimes also considered a proxy for anxiety (Fernandez Espejo, 1997; Ennaceur, 2014), was consistent between CSD and control subjects $(t=0.006, P=0.9950)$.

Together, these results suggest that despite no observed change in anxiety, novelty-driven exploration is impaired following CSD, while fear-driven exploration is comparatively resistant. To our knowledge, this is the first direct demonstration of drive-specific differences within the amotivation pathophenotype associated with social stress. 


\section{CSD does not induce deficits in short-term memory}

We next wondered whether the reduced drive to explore novel, safe space in CSD animals might lead to learning deficits, since the motivation to explore is tightly linked to learning at the molecular and cellular level (Mun et al., 2015) and episodic memory is impaired in recurrent MDD (Rapp et al., 2005). CSD-treated and control mice were therefore examined in a displaced and novel object recognition task to assess spatial and non-spatial learning.

During the habituation phase of the object recognition experiment, we found that CSD-treated subjects spent less time walking $(t=2.712, P=0.0078)$ and more time immobile $(t=3.127, P=0.0023)$ compared to the control group (Fig. 4A), consistent with the activity assay (Fig. 1E,F) and hole board test (Fig. 3A). Object interaction time, however, was not affected by CSD (Fig. 4B).

In the next phase of the experiment, subjects were returned to the identical arena following a 2 min interval during which the experimenter displaced one of the three identical objects to the opposing corner. Here we found no significant differences between groups for all the examined behaviours (Fig. 4C), and both groups demonstrated preference for the displaced object (Fig. 4D; control: $t=2.816, P=0.0073$; CSD: $t=2.843, P=0.0068)$. Similarly, in the next phase, which examines non-spatial, non-hippocampus-dependent learning, no difference in the examined behaviours were found (Fig. 4E) and both groups showed preference for the novel object (Fig. 4F; control: $t=5.098, P<0.0001$; CSD: $t=8.951, P<0.0001)$. Short-term learning and memory was therefore not affected by CSD, despite a strong deficit in the drive to explore novel, safe space. 


\section{Discussion}

Better understanding the behavioural, circuit and molecular phenotypes of MDD holds promise for the development of superior treatment strategies, including both pharmaco and psychotherapeutic interventions. In this study, we uncover the specific sensitivity of novelty-driven exploration to the CSD behavioural manipulation in mice, thereby expanding the ensemble of affected mental processes in CSD-treated animals that resemble MDD core symptomatology. At the same time, we show that fear-driven exploration is retained, providing insight into the precise nature of amotivation associated with social stress. Moreover, we show that the exploratory drive deficit is neither concurrent with anxiogenesis, nor poorer short-term memory, at least not with the behavioural paradigms employed here.

\section{CSD, neophobia and anxiety}

In the NFT, the 15 min period of scoring new frontier exploration begins immediately after the first exploration event, and therefore after a potential initiate phase of anxiety due to the conflict of avoiding versus approaching (or exploring versus not exploring) the new frontiers whilst in the homecage (McNaughton \& Gray, 2000). This component of the NFT is therefore similar to mouse homecage emergence tests, in which it has been demonstrated that anxiolytic drugs reliably decrease the latency to emerge from the homecage into an open field, and increase exploration of the latter (Jain et al., 2012). The black 6 mouse strain used in this study has relatively low trait anxiety, e.g. does not exhibit unconditioned spatial neophobia and prefers novel environments over those that are familiar (Griebel et al., 1993). Following CSD however, the black 6 mice used in this study exhibited an increased latency to first exploration events in the dark and dim versions of the NFT, which could be due to increased neophobia and resultant anxiety, in addition to a decreased motivation to explore. The hippocampus (McNaughton \& Gray, 2000) and amygdala (Tye et al., 2011) would likely contribute to such a neophobic anxiety mechanism, and both the hippocampus and amygdala show transcriptional regulation following the CSD manipulation (Azzinnari et al., 
2014). However, further research is needed to understand if the CSD manipulation used here gives rise to neophobia. Moreover, anxiogenic phenotypes in CSD-treated mice are not as robust or reproducible as those that more closely resemble specific core symptoms of MDD, including anhedonia (Tye et al., 2013) and learned helplessness (Azzinnari et al., 2014), or other MDDrelevant behavioural phenotypes including increased passive avoidance during social proximity of an aggressor mouse (Krishnan et al., 2007; Savignac et al., 2011; Holubova et al., 2014) and reduced social engagement and impaired social memory (Haque et al., 2012).

In addition, while the classical CSD manipulation commonly elicits anxiogenic-like effects in the EPM (Haque et al., 2012; Venzala et al., 2013; Kovalenko et al., 2014; Brachman et al., 2015), we did not observe any here. This was not because the CSD manipulation led to a segregation of subjects into susceptible and resilient groups, since the data were found to be normally distributed (Shapiro-Wilk normality test: CSD: $W=0.9773, P=0.8414$; Control: $W=0.9733 ; P=0.7476)$. Instead, the specific methodology exercised during the actual CSD episodes may result in a less anxious phenotype. In contrast to the CSD protocols used to date in other laboratories, we have refined the method to minimise physical injury and therefore increase the validity of CSD as a strictly psychosocial stressor (Azzinnari et al., 2014; Fuertig et al., 2015). All studies that we could find that reported anxiogenesis using the EPM did not clip the incisor teeth of the CD1 aggressor males (e.g. (Haque et al., 2012; Venzala et al., 2013; Kovalenko et al., 2014; Brachman et al., 2015)). Aggression bursts in these studies could rapidly inflict physical wounds on the black 6 subordinate males, even if the experimenter terminated or interrupted the wound-inflicting attack, and may strongly influence the specific ensemble of resulting behavioural phenotypes. Therefore, while we cannot rule out neophobia, we can state that we find no evidence for it, and consider it to be an unlikely finding, especially considering the mouse strain used and a clear lack of an anxiogenic effect in the hole-board task (no change in head-dip or grooming time) and EPM.

We did however observe that CSD-treated subjects exhibited reduced ambulation selectively in the closed and dimly lit arms of the EPM, a finding that foreshadowed our subsequent experiments 
on exploratory behaviour and hinted towards selectivity amongst the amotivation pathophenotype associated with social stress.

\section{CSD, MDD and exploratory drive}

In the post-CSD activity assay, we found a reduction in rearing in CSD-treated subjects compared to both the control group, and the CSD group pre-CSD (Fig. 3G). In contrast, measures of immobility and horizontal ambulation only demonstrated between-subject significance. This greater sensitivity of rearing to CSD has also been observed by others (Haque et al., 2012), and suggests CSD may induce exploration deficits. However, the activity assay provided no insight into the potential underlying motivational basis for this deficit in rearing.

Using the NFT, we were able to dissociate between novelty-driven and fear-driven exploration (Saab et al., 2009; Mun et al., 2015). Surprisingly, we found that CSD-treated subjects demonstrated a marked deficit in exploring new frontiers exclusively when their homecage was illuminated with either red or dim white light. We interpret this finding as a specific impairment in novelty-driven exploration since alternative motivational factors, such as fear, hunger, thirst, and the desire to mate were absent or minimised. In contrast, when the NFT was performed with the homecage brightly illuminated with white light, we found no statistical significance between CSDtreated subjects and controls. These findings fit well with clinical observations in patients with MDD, who demonstrate amotivation (Calabrese et al., 2014) and decreased exploratory excitability (Bensaeed et al., 2014; Zaninotto et al., 2016), but a normal, or even heightened, unconditioned fear response (Dixon-Gordon et al., 2015).

The specific deficit that we find in novelty-driven exploration provides insight into what brain areas underlie amotivation in rodents that undergo CSD, particularly implicating the nucleus accumbens (Lever et al., 2006) and hippocampus (Saab et al., 2009; Mun et al., 2015). The nucleus accumbens regulates motivation in mammals (Da Cunha et al., 2012), is essential for rearing (Lever et al., 2006), and CSD induces spine morphology alterations and increased synaptic transmission 
amongst medium spiny inhibitory neurons in this area (Christoffel et al., 2011). In addition, we have demonstrated that CSD and dopamine depletion in the nucleus accumbens both lead to reduced motivation to obtain food reward under effortful conditions (Bergamini et al., 2016). These findings and others have made the nucleus accumbens a new potential target area to treat MDD through deep-brain stimulation (Nauczyciel et al., 2013), and raise the possibility that structural modifications within the nucleus accumbens, potentially instigated from hippocampo-amygdalar signalling, underlie the blunted drive to explore observed in this study.

Indeed, circuitry underlying novelty-driven exploration may be particularly susceptible to disruption following CSD. The hippocampus-dependent detection of spatial novelty is sometimes impaired following CSD (Howard et al., 2011; Kaplan et al., 2014), and hippocampus-dependent detection of spatial novelty is instrumental for novelty-driven exploration (Saab et al., 2009; Mun et al., 2015), which we show here is markedly impaired following CSD. Moreover, we have previously reported that the CSD manipulation results in transcriptional changes in the hippocampus (Azzinnari et al., 2014). Clinically, hippocampal dysfunction is one of the most commonly reported neuropathophysiological observations in MDD (Wainwright \& Galea, 2013; Fasick et al., 2015; Malykhin \& Coupland, 2015), and may be both a contributing factor in MDD aetiology, as well as a potential target for therapy.

For example, re-enforcing exploration in MDD patients might prove therapeutic for other elements of the illness by facilitating normal function of the hippocampus and/or initiating a positive-feedback loop whereby renewed exploration events give rise to positive outcomes that serve to re-enforce further exploration and more positive outcomes. Novel paradigms, like the NFT, combined with robust animal manipulations that give rise to symptomatology reminiscent of MDD, like the CSD manipulation, will therefore be essential in guiding the development of treatments for mental illness along these lines.

\section{CSD and spatial memory}


We found no evidence for a cognitive deficit in spatial and non-spatial learning and memory using a displaced and novel object recognition task. This was somewhat surprising given the profound deficit in exploratory drive following the CSD manipulation and the importance of volitional exploration and rearing for efficient spatial learning (Mun et al., 2015). It is possible that the shortterm task employed in the current study was not sufficiently demanding for potential CSD-induced deficits in memory to emerge. Indeed, using a complex reversal learning operant reward test, we recently found that the same CSD manipulation led to impairments (Bergamini et al., 2016), demonstrating that cognitive abilities are affected.

Ideally, to fully understand the relationship between exploratory drive and cognitive deficits following CSD, detailed experiments that examine learning and memory in conjunction with hippocampal place cell formation and other neural correlated of memory are needed. 


\section{Conclusion}

Amotivation is a core symptom of human depression and is commonly found in animals that have experienced prolonged psychosocial stress. Here we show that the amotivation pathophenotype in mice following chronic social defeat diverged across different motivators. Specifically, noveltydrive exploration was severely impaired, while fear-driven exploration remained intact.

Importantly, the reduced volitional exploration of novel, safe space was not associated with anxiogenesis and did not lead to impairments in short-term memory. This study therefore increases our resolution into the amotivation phathophenotype associated with psychosocial stress, highlighting potential for new avenues of research and treatment. 


\section{Acknowledgements}

We are grateful to Damiano Azzinnari, Hannes Sigrist and Giorgio Bergamini for technical assistance and helpful discussions. ATC and BJS designed and performed experiments. ATC, HIC and BJS analysed data. All authors discussed the data, contributed to the manuscript and declare no conflicts of interests.

Funding: This work was supported by the Swiss National Science Foundation (PZ00P3_148114/1), the University of Zurich (FK-13-048), the Brain and Behavior Research Foundation (19213) and the Neuroscience Center Zurich through funding to BJS. 


\section{References}

Aghajan, Z.M., Acharya, L., Moore, J.J., Cushman, J.D., Vuong, C. \& Mehta, M.R. (2015) Impaired spatial selectivity and intact phase precession in two-dimensional virtual reality. Nat Neurosci, 18, 121-128.

Archer, J. (1973) Tests for emotionality in rats and mice: a review. Animal behaviour, 21, 205-235.

Azzinnari, D., Sigrist, H., Staehli, S., Palme, R., Hildebrandt, T., Leparc, G., Hengerer, B., Seifritz, E. \& Pryce, C.R. (2014) Mouse social stress induces increased fear conditioning, helplessness and fatigue to physical challenge together with markers of altered immune and dopamine function. Neuropharmacology, 85, 328-341.

Bensaeed, S., Ghanbari Jolfaei, A., Jomehri, F. \& Moradi, A. (2014) Comparison of Temperament and Character in Major Depressive Disorder Versus Bipolar II Disorder. Iranian journal of psychiatry and behavioral sciences, 8, 28-32.

Bergamini, G., Cathomas, F., Auer, S., Sigrist, H., Seifritz, E., Patterson, M., Gabriel, C., Pryce, C.R. (2016) Mouse psychosocial stress reduces motivation and cognitive function in operant reward tests: A model for reward pathology with effects of agomelatine. European Neuropsychopharmacology, 26, 1448-1464.

Blanchard, R.J., McKittrick, C.R. \& Blanchard, D.C. (2001) Animal models of social stress: effects on behavior and brain neurochemical systems. Physiology \& behavior, 73, 261-271. 
Brachman, R.A., McGowan, J.C., Perusini, J.N., Lim, S.C., Pham, T.H., Faye, C., Gardier, A.M., Mendez-David, I., David, D.J., Hen, R. \& Denny, C.A. (2015) Ketamine as a Prophylactic Against Stress-Induced Depressive-Like Behavior. Biol Psychiatry.

Calabrese, J.R., Fava, M., Garibaldi, G., Grunze, H., Krystal, A.D., Laughren, T., Macfadden, W., Marin, R., Nierenberg, A.A. \& Tohen, M. (2014) Methodological approaches and magnitude of the clinical unmet need associated with amotivation in mood disorders. Journal of affective disorders, 168, 439-451.

Christoffel, D.J., Golden, S.A., Dumitriu, D., Robison, A.J., Janssen, W.G., Ahn, H.F., Krishnan, V., Reyes, C.M., Han, M.H., Ables, J.L., Eisch, A.J., Dietz, D.M., Ferguson, D., Neve, R.L., Greengard, P., Kim, Y., Morrison, J.H. \& Russo, S.J. (2011) IkappaB kinase regulates social defeat stress-induced synaptic and behavioral plasticity. J Neurosci, 31, 314-321.

Da Cunha, C., Gomez, A.A. \& Blaha, C.D. (2012) The role of the basal ganglia in motivated behavior. Rev Neurosci, 23, 747-767.

Der-Avakian, A. \& Markou, A. (2012) The neurobiology of anhedonia and other reward-related deficits. Trends Neurosci, 35, 68-77.

Dixon-Gordon, K.L., Weiss, N.H., Tull, M.T., DiLillo, D., Messman-Moore, T. \& Gratz, K.L. (2015) Characterizing emotional dysfunction in borderline personality, major depression, and their co-occurrence. Comprehensive psychiatry, 62, 187-203. 
DSM-5 (2013) Diagnostic and statistical manual of mental disorders. Revision American Psychiatric Association. Washington, DC, fifth ed. American Psychiatric Association, Washington, DC.

Ennaceur, A. (2014) Tests of unconditioned anxiety - pitfalls and disappointments. Physiology \& behavior, 135, 55-71.

Fasick, V., Spengler, R.N., Samankan, S., Nader, N.D. \& Ignatowski, T.A. (2015) The hippocampus and TNF: Common links between chronic pain and depression. Neurosci Biobehav Rev, 53, 139-159.

Fernandez Espejo, E. (1997) Structure of the mouse behaviour on the elevated plus-maze test of anxiety. Behav Brain Res, 86, 105-112.

File, S.E. \& Wardill, A.G. (1975) Validity of head-dipping as a measure of exploration in a modified hole-board. Psychopharmacologia, 44, 53-59.

Fuertig, R., Azzinnari, D., Bergamini, G., Cathomas, F., Sigrist, H., Seifritz, E., Vavassori, S., Luippold, A., Hengerer, B., Ceci, A. \& Pryce, C.R. (2015) Mouse chronic social stress increases blood and brain kynurenine pathway activity and fear behaviour: Both effects are reversed by inhibition of indoleamine 2,3-dioxygenase. Brain, behavior, and immunity.

Gomez, R.G., Fleming, S.H., Keller, J., Flores, B., Kenna, H., DeBattista, C., Solvason, B. \& Schatzberg, A.F. (2006) The neuropsychological profile of psychotic major depression and its relation to cortisol. Biol Psychiatry, 60, 472-478. 
Grandjean, J., Azzinnari, D., Seuwen, A. Sigrist H., Seifritz, E., Pryce, C.R. \& Rudin, M. (2016) Chronic psychosocial stress in mice leads to changes in brain functional connectivity and metabolite levels comparable to human depression. Neuroimage, Epub ahead of print.

Griebel, G., Belzung, C., Misslin, R. \& Vogel, E. (1993) The free-exploratory paradigm: an effective method for measuring neophobic behaviour in mice and testing potential neophobiareducing drugs. Behav Pharmacol, 4, 637-644.

Gustafsson, L. \& Paplinski, A.P. (2004) Self-organization of an artificial neural network subjected to attention shift impairments and familiarity preference, characteristics studied in autism. Journal of autism and developmental disorders, 34, 189-198.

Haque, F.N., Lipina, T.V., Roder, J.C. \& Wong, A.H. (2012) Social defeat interacts with Disc1 mutations in the mouse to affect behavior. Behav Brain Res, 233, 337-344.

Harvey, P.D. (2007) Cognitive impairments in major depression and bipolar disorders. Psychiatry, 4, 12-14.

Hershenberg, R., Satterthwaite, T.D., Daldal, A., Katchmar, N., Moore, T.M., Kable, J.W. \& Wolf, D.H. (2016) Diminished effort on a progressive ratio task in both unipolar and bipolar depression. Journal of affective disorders, 196, 97-100.

Holubova, K., Nekovarova, T., Pistovcakova, J., Sulcova, A., Stuchlik, A. \& Vales, K. (2014) Pregnanolone glutamate, a novel use-dependent NMDA receptor inhibitor, exerts antidepressantlike properties in animal models. Front Behav Neurosci, 8. 
Howard, L.R., Kumaran, D., Olafsdottir, H.F. \& Spiers, H.J. (2011) Double dissociation between hippocampal and parahippocampal responses to object-background context and scene novelty. $J$ Neurosci, 31, 5253-5261.

Jain, A., Dvorkin, A., Fonio, E., Golani, I. \& Gross, C.T. (2012) Validation of the dimensionality emergence assay for the measurement of innate anxiety in laboratory mice. European neuropsychopharmacology : the journal of the European College of Neuropsychopharmacology, 22, 153-163.

Kaplan, R., Horner, A.J., Bandettini, P.A., Doeller, C.F. \& Burgess, N. (2014) Human hippocampal processing of environmental novelty during spatial navigation. Hippocampus, 24, 740-750.

Karl, T., Pabst, R. \& von Horsten, S. (2003) Behavioral phenotyping of mice in pharmacological and toxicological research. Experimental and toxicologic pathology : official journal of the Gesellschaft fur Toxikologische Pathologie, 55, 69-83.

Kesner, R.P. \& Hopkins, R.O. (2006) Mnemonic functions of the hippocampus: a comparison between animals and humans. Biological psychology, 73, 3-18.

Kovalenko, I.L., Galyamina, A.G., Smagin, D.A., Michurina, T.V., Kudryavtseva, N.N. \& Enikolopov, G. (2014) Extended effect of chronic social defeat stress in childhood on behaviors in adulthood. PLoS One, 9, e91762.

Krishnan, V., Han, M.H., Graham, D.L., Berton, O., Renthal, W., Russo, S.J., Laplant, Q., Graham, A., Lutter, M., Lagace, D.C., Ghose, S., Reister, R., Tannous, P., Green, T.A., Neve, R.L., Chakravarty, S., Kumar, A., Eisch, A.J., Self, D.W., Lee, F.S., Tamminga, C.A., Cooper, D.C., 
Gershenfeld, H.K. \& Nestler, E.J. (2007) Molecular adaptations underlying susceptibility and resistance to social defeat in brain reward regions. Cell, 131, 391-404.

Langston, R.F., Stevenson, C.H., Wilson, C.L., Saunders, I. \& Wood, E.R. (2010) The role of hippocampal subregions in memory for stimulus associations. Behav Brain Res, 215, 275-291.

Lever, C., Burton, S. \& O'Keefe, J. (2006) Rearing on hind legs, environmental novelty, and the hippocampal formation. Rev Neurosci, 17, 111-133.

Malykhin, N.V. \& Coupland, N.J. (2015) Hippocampal neuroplasticity in major depressive disorder. Neuroscience.

McNaughton, N. \& Gray, J.A. (2000) Anxiolytic action on the behavioural inhibition system implies multiple types of arousal contribute to anxiety. Journal of affective disorders, 61, 161176.

Mun, H.S., Saab, B.J., Ng, E., McGirr, A., Lipina, T.V., Gondo, Y., Georgiou, J. \& Roder, J.C. (2015) Self-directed exploration provides a Ncs1-dependent learning bonus. Scientific reports, 5, 17697.

Nauczyciel, C., Robic, S., Dondaine, T., Verin, M., Robert, G., Drapier, D., Naudet, F. \& Millet, B. (2013) The nucleus accumbens: a target for deep brain stimulation in resistant major depressive disorder. Journal of molecular psychiatry, 1, 17.

Pellow, S., Chopin, P., File, S.E. \& Briley, M. (1985) Validation of open:closed arm entries in an elevated plus-maze as a measure of anxiety in the rat. J Neurosci Methods, 14, 149-167. 
Pryce, C.R., Azzinnari, D., Sigrist, H., Gschwind, T., Lesch, K.P. \& Seifritz, E. (2012) Establishing a learned-helplessness effect paradigm in C57BL/6 mice: behavioural evidence for emotional, motivational and cognitive effects of aversive uncontrollability per se. Neuropharmacology, $6 \mathbf{2}$, 358-372.

Rapp, M.A., Dahlman, K., Sano, M., Grossman, H.T., Haroutunian, V. \& Gorman, J.M. (2005) Neuropsychological differences between late-onset and recurrent geriatric major depression. The American journal of psychiatry, 162, 691-698.

Rygula, R., Abumaria, N., Flugge, G., Fuchs, E., Ruther, E. \& Havemann-Reinecke, U. (2005) Anhedonia and motivational deficits in rats: impact of chronic social stress. Behav Brain Res, 162, 127-134.

Saab, B.J., Georgiou, J., Nath, A., Lee, F.J., Wang, M., Michalon, A., Liu, F., Mansuy, I.M. \& Roder, J.C. (2009) NCS-1 in the dentate gyrus promotes exploration, synaptic plasticity, and rapid acquisition of spatial memory. Neuron, 63, 643-656.

Savignac, H.M., Hyland, N.P., Dinan, T.G. \& Cryan, J.F. (2011) The effects of repeated social interaction stress on behavioural and physiological parameters in a stress-sensitive mouse strain. Behav Brain Res, 216, 576-584.

Strauss, G.P., Frank, M.J., Waltz, J.A., Kasanova, Z., Herbener, E.S. \& Gold, J.M. (2011) Deficits in positive reinforcement learning and uncertainty-driven exploration are associated with distinct aspects of negative symptoms in schizophrenia. Biol Psychiatry, 69, 424-431. 
Takeda, H., Tsuji, M., Matsumiya, T. (1998) Changes in head-dipping behaviour in the hole-board test reflect the anxiolytic sate in mice. European Journal of Pharmacology, 350, 21-29.

Torgersen, S., Onstad, S., Skre, I., Edvardsen, J. \& Kringlen, E. (1993) "True" schizotypal personality disorder: a study of co-twins and relatives of schizophrenic probands. The American journal of psychiatry, 150, 1661-1667.

Tsai Cabal, A., Ioanas, H-I., Seifritz, E., Pryce, C.R., Saab, B.J. (2016) Mouse Housing Population Size Does not Affect Performance in the New Frontier Task. Matters, in press.

Tye, K.M., Mirzabekov, J.J., Warden, M.R., Ferenczi, E.A., Tsai, H.C., Finkelstein, J., Kim, S.Y., Adhikari, A., Thompson, K.R., Andalman, A.S., Gunaydin, L.A., Witten, I.B. \& Deisseroth, K. (2013) Dopamine neurons modulate neural encoding and expression of depression-related behaviour. Nature, 493, 537-541.

Tye, K.M., Prakash, R., Kim, S.Y., Fenno, L.E., Grosenick, L., Zarabi, H., Thompson, K.R., Gradinaru, V., Ramakrishnan, C. \& Deisseroth, K. (2011) Amygdala circuitry mediating reversible and bidirectional control of anxiety. Nature, 471, 358-362.

Venzala, E., Garcia-Garcia, A.L., Elizalde, N. \& Tordera, R.M. (2013) Social vs. environmental stress models of depression from a behavioural and neurochemical approach. European neuropsychopharmacology : the journal of the European College of Neuropsychopharmacology, 23, 697-708.

Vorhees, C.V. \& Williams, M.T. (2014) Assessing spatial learning and memory in rodents. ILAR journal / National Research Council, Institute of Laboratory Animal Resources, 55, 310-332. 
Wainwright, S.R. \& Galea, L.A. (2013) The neural plasticity theory of depression: assessing the roles of adult neurogenesis and PSA-NCAM within the hippocampus. Neural plasticity, 2013, 805497.

Williams, E., Costall, A. \& Reddy, V. (1999) Children with autism experience problems with both objects and people. Journal of autism and developmental disorders, 29, 367-378.

Yan, H.C., Cao, X., Das, M., Zhu, X.H. \& Gao, T.M. (2010) Behavioral animal models of depression. Neuroscience bulletin, 26, 327-337.

Zaninotto, L., Solmi, M., Toffanin, T., Veronese, N., Cloninger, C.R. \& Correll, C.U. (2016) A meta-analysis of temperament and character dimensions in patients with mood disorders: Comparison to healthy controls and unaffected siblings. Journal of affective disorders, 194, 8497. 


\section{Figure Legends}

Fig. 1. CSD reduced bodyweight homeostasis, ambulation and rearing, but was not anxiogenic. (A) Study design consisted of handling, 15 days of CSD and a behavioral battery. (B) CSD-treated subjects had increased daily bodyweight fluctuation. (C) Both CSD-treated and control mice spent less time in the open arms of the EPM compared to the closed arms and no between-group differences were observed. (D) CSD-treated subjects specifically showed reduced ambulation in the closed arms of the EPM. (E-D) In an activity assay, CSD-treated mice demonstrated (E) less horizontal ambulation, (F) more immobility and (G) reduced rearing, compared to the control group. In addition, control animals showed an (E) increase in horizontal ambulation in the second activity assay that was not observed in the CSD-treated group, while the CSD-treated group showed a $(G)$ reduction in rearing in the second activity assay that was not observed in the control group.

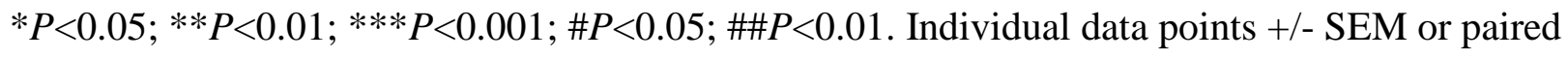
individual data points shown.

Fig. 2. CSD specifically affected novelty-driven exploration. (A) New frontier task (NFT) apparatus, showing the dark, dim and bright versions. (B) The latency to the first exploration event was larger for CSD-treated mice in the dark version. (C) The latency to the second exploration event was larger for CSD-treated mice in the dark and dim versions. (D) No differences in latency were observed for exploration to all four frontiers when corrected for multiple comparisons. (E-G) In the safe (dark and dim) versions of the NFT, CSD-treated mice demonstrated fewer exploration events throughout the experiment. However, no differences were found in the fearful (bright) version of the task. $* P<0.05 ; * * P<0.01 ; * * * P<0.001 ; \# P<0.05$. Group mean $+/-$ SEM shown.

Fig. 3. CSD-treated animals exhibited elevated immobility in the hole board test. (A) Analyzed behaviours. (B) No difference in head dips between the groups. $* * P<0.01$. Group mean or individual data points +/- SEM shown. 
Fig. 4. CSD does not affect short-term learning and memory. (A) Analyzed behaviours and (B) object interaction time during the habituation phase. (C) Analyzed behaviors and (D) object interaction time during the displaced object phase. (E) Analyzed behaviors and (F) object interaction time during the novel object phase. $* * P<0.01$; **** $P<0.0001$. Group mean $+/-$ SEM shown. 
Figure 1

A

Time (day)

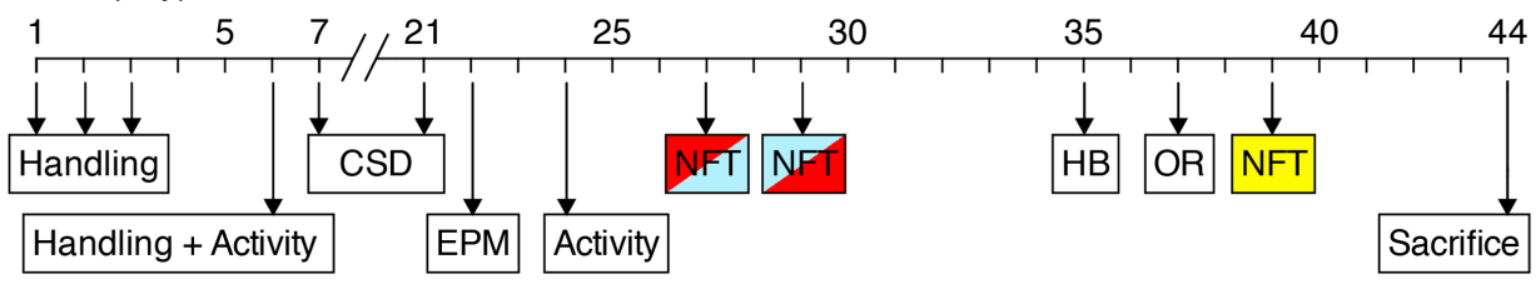

B

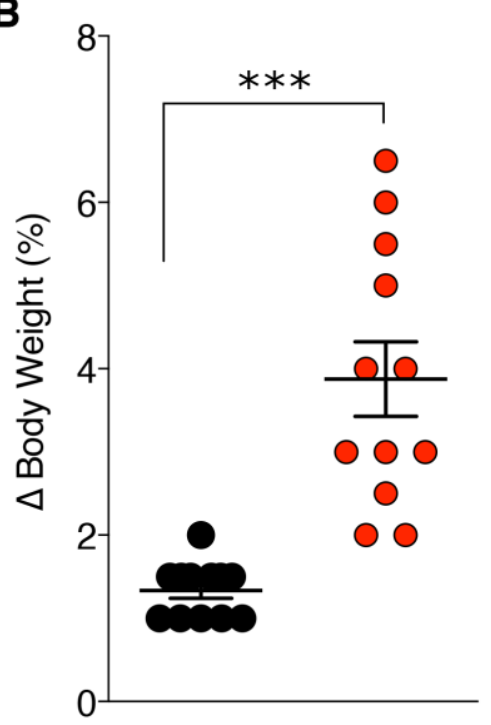

C

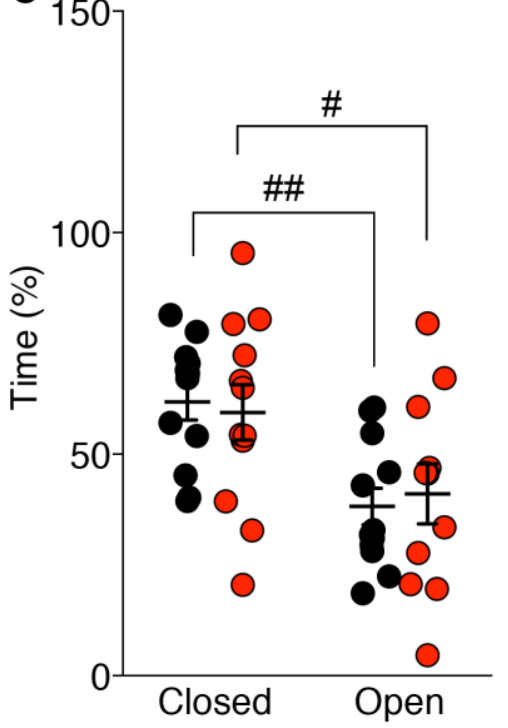

E

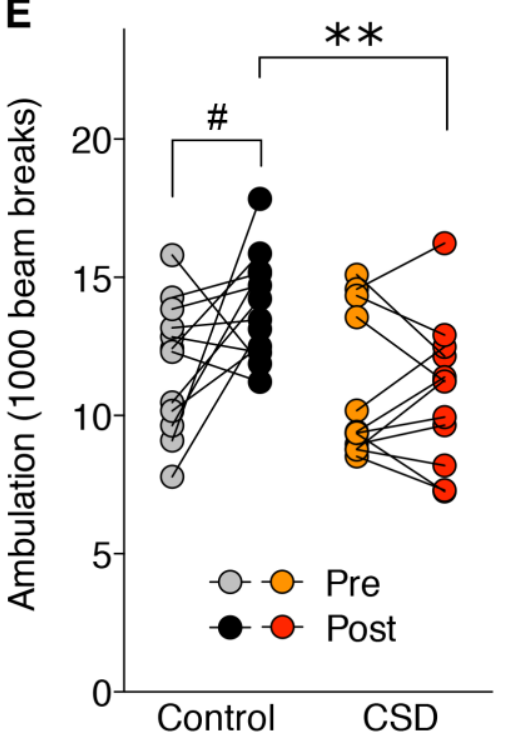

F

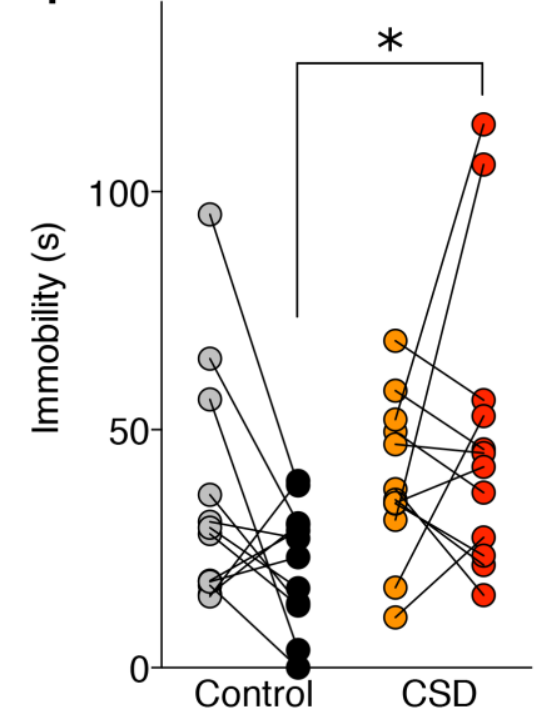

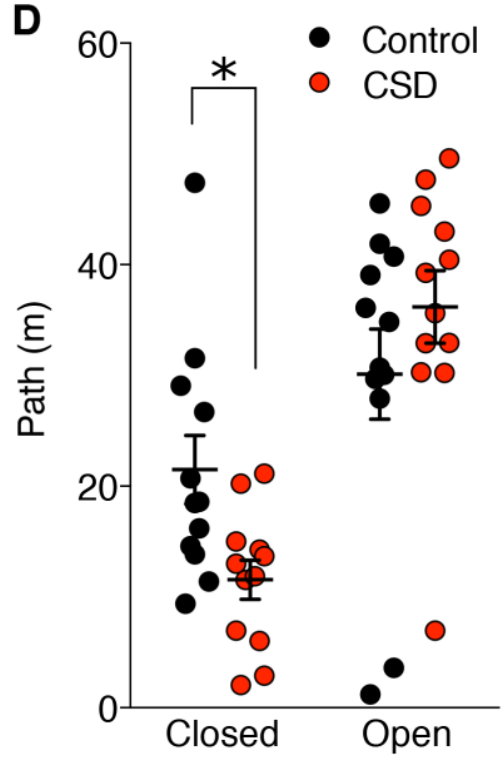

G

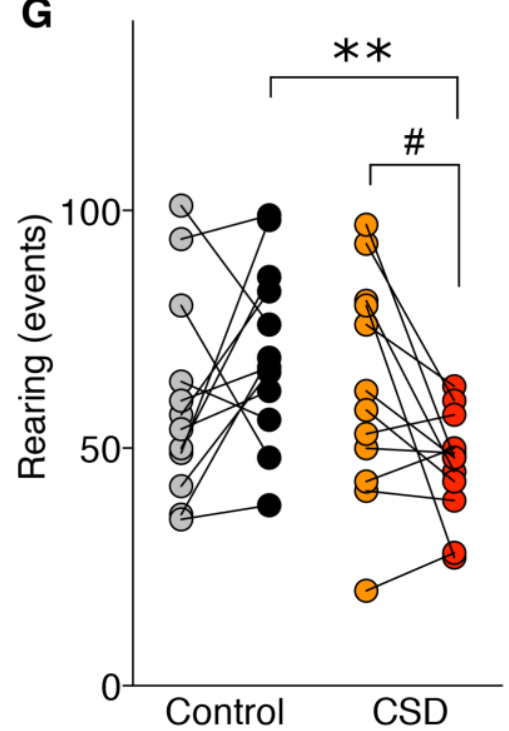


Figure 2

A New Frontier Task (NFT) with various illuminations

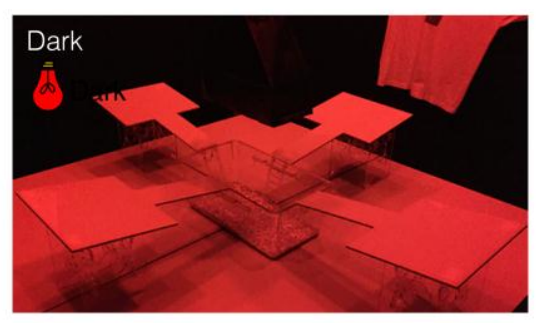

B First Frontier

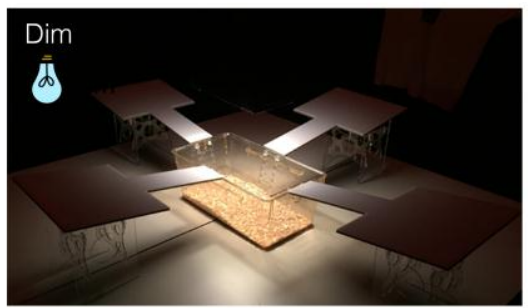

C

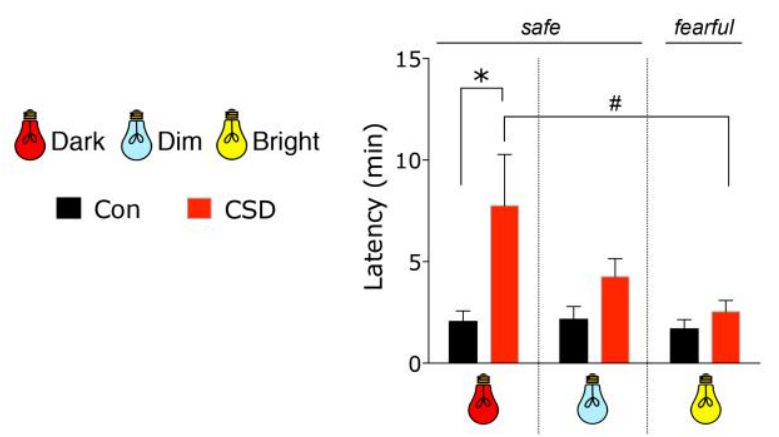

E

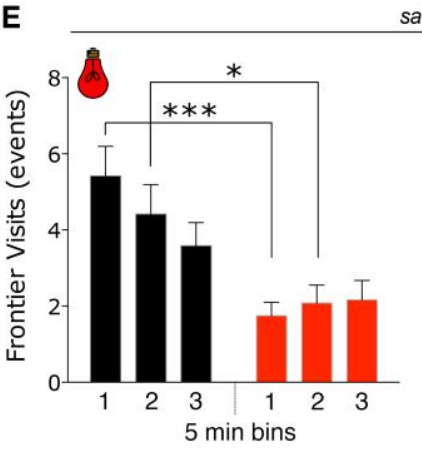

safe

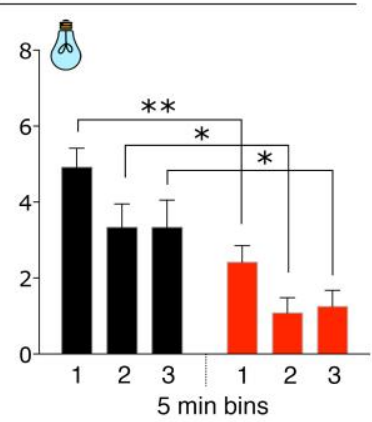

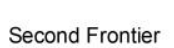

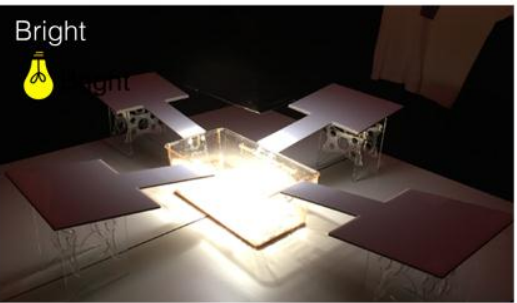

D $_{\text {Forth Frontier }}$

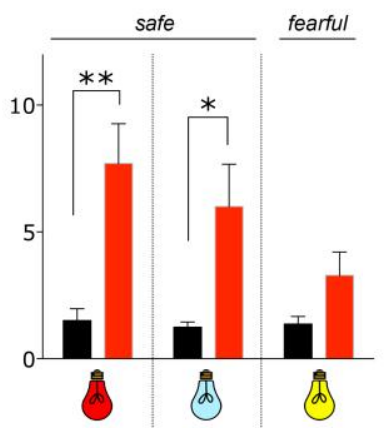

$\mathbf{F}$

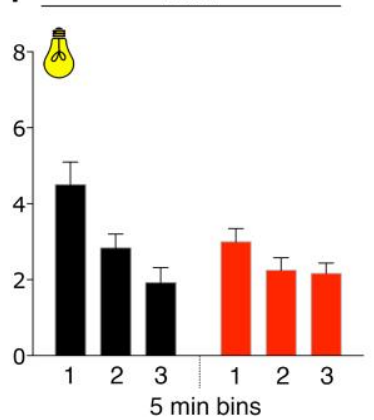

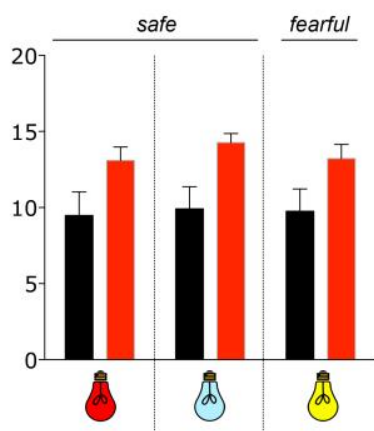

G
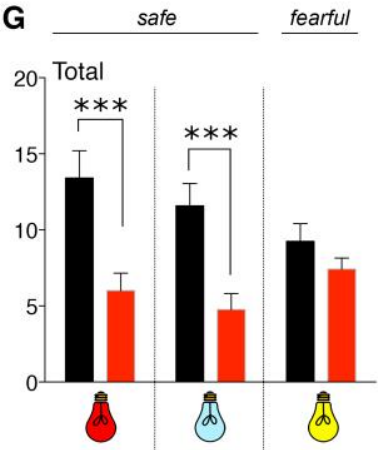
Figure 3

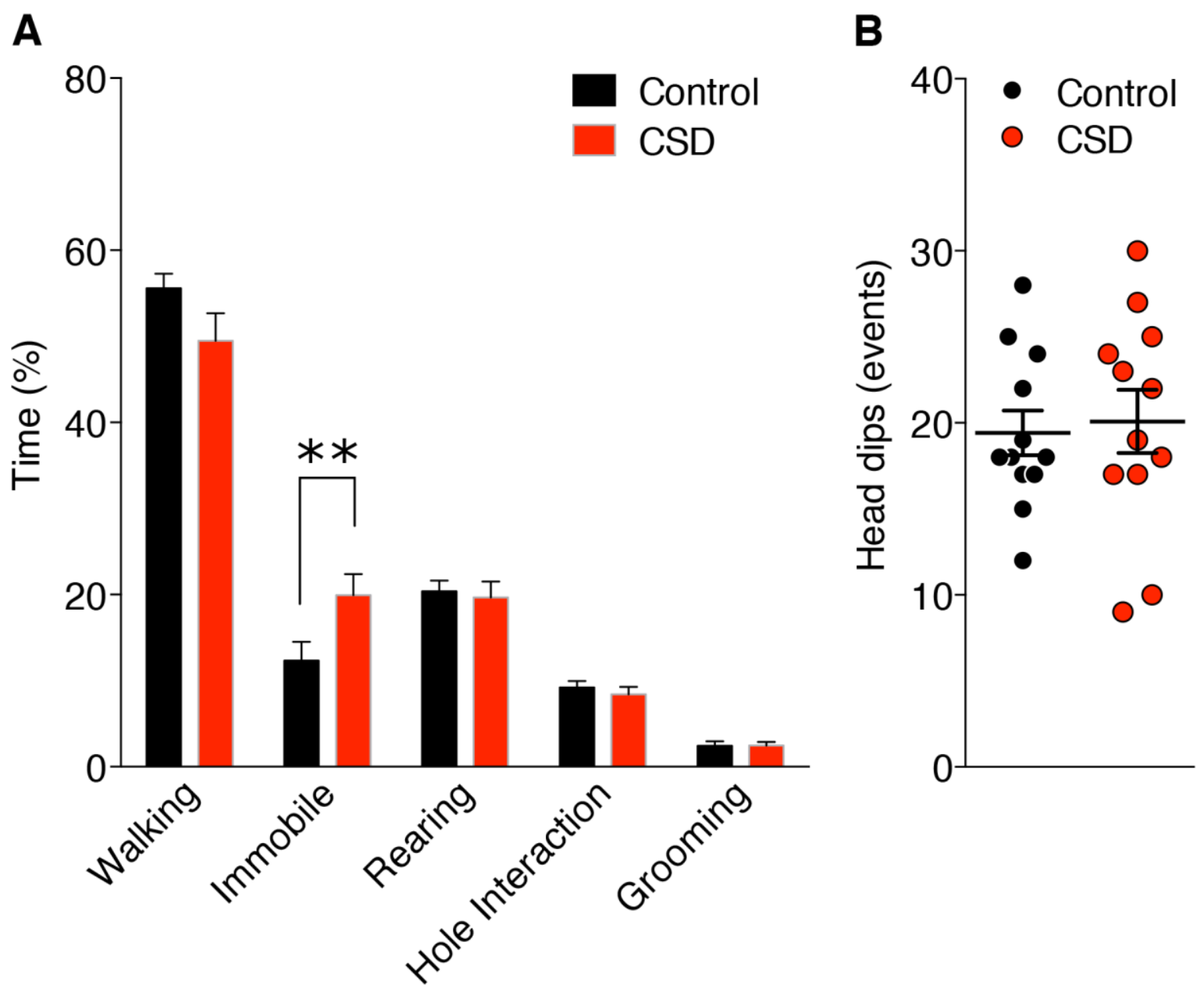


Figure 4
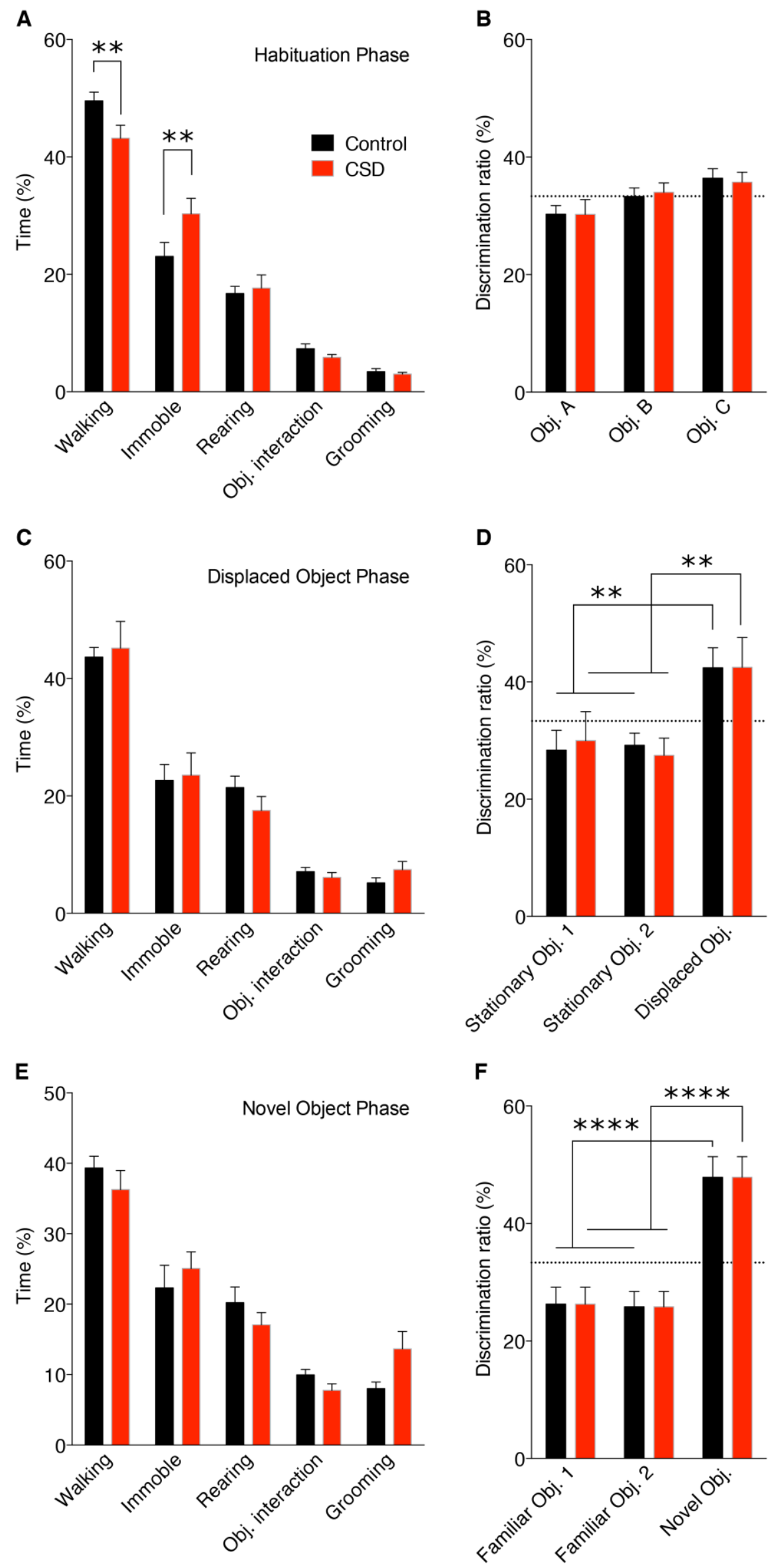
ACSD Acontiol

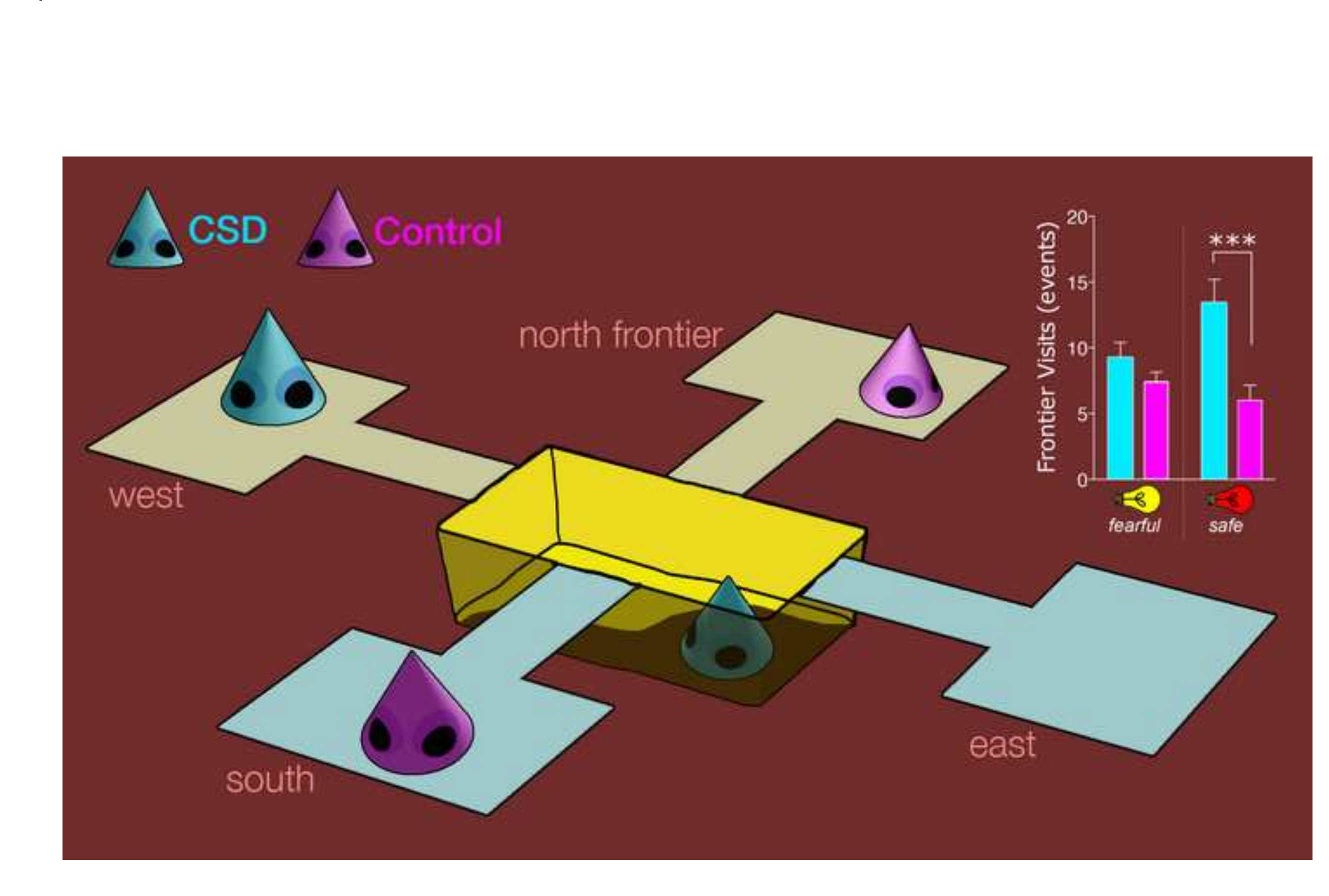

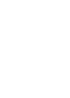

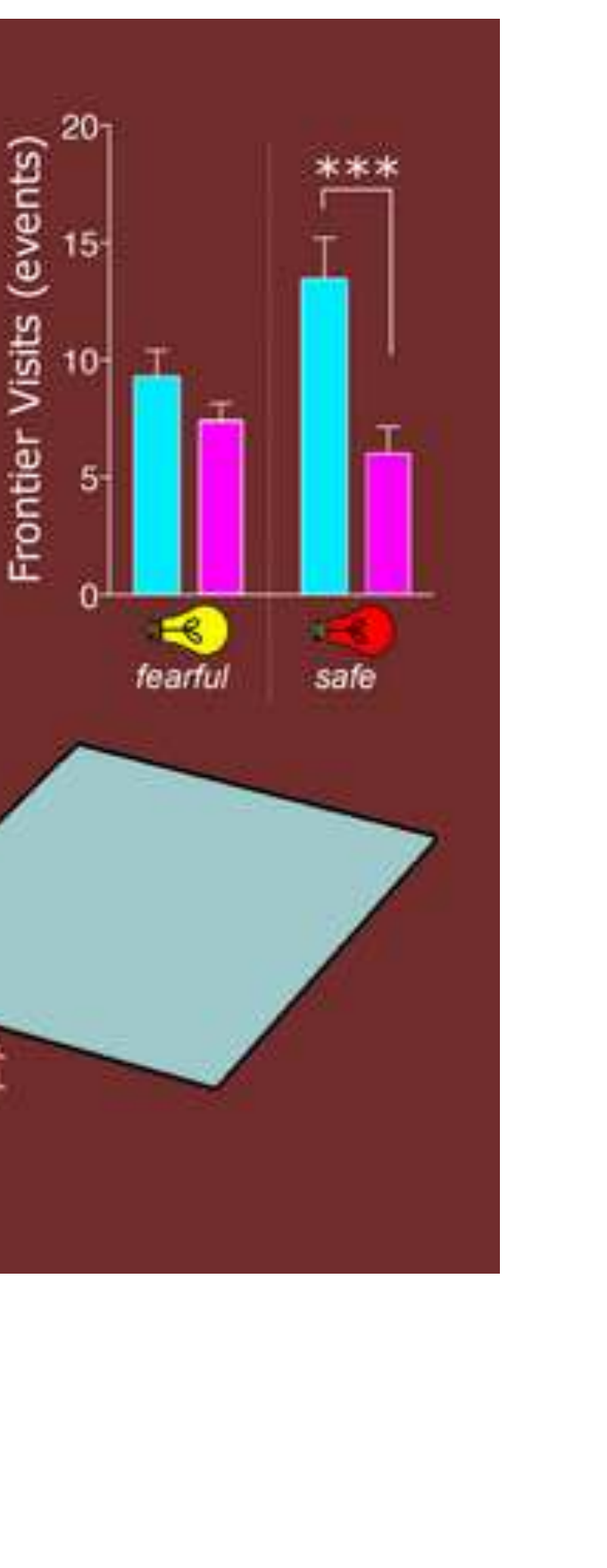

